\title{
Resolución experimental y analítica de un problema de dinámica rotacional: interpretación de los resultados ${ }^{+*}$
}

\author{
Beatriz Follari ${ }^{1}$ \\ Gilda Dima ${ }^{2}$ \\ Departamento de Física \\ Universidad Nacional de La Pampa \\ Santa Rosa - Argentina
}

\section{Resumen}

La resolución de problemas y las prácticas de laboratorio son parte esencial de las actividades que deben realizar los estudiantes de Física en sus primeros cursos de Física. Se relata una experiencia de aula en la que se propuso a los estudiantes de Física II del primer año de las carreras Profesorado y Licenciatura en Física, el estudio de un carrete que rueda sin deslizar. La misma constó de dos partes: en la primera se les solicitó que planificaran y llevaran a cabo una experiencia para determinar el momento de inercia del carrete; en la segunda se les pidió el desarrollo analítico para encontrar la aceleración y fuerza de rozamiento al hacerlo rodar. La actividad se llevó a cabo en grupo y los resultados fueron expuestos y discutidos. La propuesta resultó positiva ya que llevó a los estudiantes a expresar sus ideas, a la discusión grupal y promovió la autonomía para planificar experiencias sencillas y llevarlas a cabo. Se puso énfasis en la interpretación de las ecuaciones resultantes.

Palabras-clave: Dinámica de rotaciones; Problemas; Tarea experimental; Trabajo en grupos.

\footnotetext{
${ }^{+}$Experiental resolution and analysis of a dynamic rotational problema: interpretation of results

* Recebido: dezembro de 2015.

Aceito: abril de 2016.

${ }^{1}$ E-mail: beatrizfollari@hotmail.com

2 E-mail: dimascari@cpenet.com.ar
} 


\begin{abstract}
Problem-solving and lab practices are essential parts in the activities Physics students have to do in their first Physics courses. A classroom experience is told in which students were asked to study a reel that rolls but does not slide. This experience was carried out by Physics II students from Profesorado and Licenciatura in Physics. This experience consisted of two main parts: in the first one, students were asked to plan and perform an experience to decide the moment of inertia of the reel; in the second one, students were asked the analytical development to find the acceleration and the friction force when the reel is rolling. The activity was developed in groups and results were presented and argued. This proposal brought about positive results since students could express their ideas and participate in a group discussion. Besides, it promoted students' autonomy to plan simple experiences and carry them out. The emphasis was on the interpretation of the resulting equations.
\end{abstract}

Keywords: Dynamics of rotations; Problems; Experiential task; Group work.

\title{
I. Introducción
}

Creemos que los futuros profesores y licenciados en Física deben ser capaces de programar y planificar actividades tanto experimentales como analíticas para solucionar situaciones físicas determinadas. Su preparación es parte de nuestra labor como docentes, debiendo comenzar con esta práctica desde los primeros años.

Entre los objetivos que debemos proponernos se encuentran los de favorecer en los estudiantes la comunicación, tanto oral como escrita, de los resultados encontrados en la experimentación y también la capacidad de explicar sus razonamientos para alguna situación de resolución matemática.

En las Físicas Básicas que cursan nuestros estudiantes de Licenciatura y Profesorado en Física de la Facultad de Ciencias Exactas y Naturales es necesario no sólo recurrir al laboratorio para que los alumnos comprendan los contenidos sino que deben aprender a realizar la actividad experimental en sí misma. Se busca que vayan adquiriendo cierta autonomía y que sean capaces de proponer experiencias sencillas. La utilización de material no convencional o de uso cotidiano les ayuda a entender los conceptos.

En general los alumnos de Física, en sus primeros años, cursan las Físicas Básicas simultáneamente con Análisis Matemático y Álgebra. En estas asignaturas los conceptos son presentados con rigor matemático pero no es tan frecuente la interpretación de resultados. El análisis de las ecuaciones a las que se arriba en la resolución de ciertos problemas presenta 
algunas dificultades. Los docentes de Física pretendemos que nuestros alumnos "vean" la contribución de las variables, qué ocurre si alguna aumenta o disminuye o se suprime. Es parte de los saberes que deben tratarse con detenimiento en las primeras físicas de la carrera.

Es por ello que redactamos una propuesta didáctica donde se intentó que la resolución fuese abordada desde lo experimental y lo analítico. El tema elegido fue: Dinámica de Rotaciones. La actividad fue desarrollada como cierre de la unidad, para reforzar no sólo los conceptos de torque, momento de inercia y rodadura sino también los vínculos entre la Física y la Matemática.

\section{Marco teórico}

Nuestras estrategias didácticas deben llevar a los estudiantes a que la información nueva no se "acumule" con la ya existente, es decir animarlos a que desarrollen capacidades que les permitan interpretar, reflexionar, relacionar y comunicar. Estas acciones propenderán también a que los nuevos conceptos puedan ser aplicados en distintos contextos y situaciones.

El trabajo en el aula debe tener en cuenta la diversidad y la heterogeneidad, generando metodologías que promuevan la interacción entre pares, la producción de trabajos de manera conjunta, la resolución de situaciones problemáticas, etc. donde cada uno de los alumnos cumpla una función determinada. Existen diversas investigaciones que dan sustento a la idea de que el trabajo en grupo favorece el aprendizaje (DAVINI, 2009; LITWIN, 2009; REDISH, 2003; SOUTO, 1996). La diversidad de las características de los integrantes del grupo beneficia el crecimiento individual.

La idea de conformar grupos de trabajo en nuestras aulas se contrapone con las características de aulas tradicionales, donde el docente es quien va construyendo la tarea y dirigiendo el razonamiento de sus alumnos. En las actividades grupales el profesor, trabajando sobre la base de las respuestas de sus estudiantes, será el encargado de incentivarlos a expresar sus ideas y a razonar sobre la base de los conceptos estudiados (COSTAMAGNA, 2005).

El trabajo de laboratorio es una actividad básica en las clases de Física; encontramos en la bibliografía distintas categorías de los mismos: tradicionales, abiertos, estructurados, etc. Es verdad que cada una de ellas presenta aspectos positivos y negativos, sin embargo en la medida de nuestras posibilidades los profesores de Física intentamos incluirlas en nuestras aulas. Las dificultades para desarrollar la tarea de laboratorio son variadas: falta de material, falta de personal de apoyo, poco tiempo para trabajar, etc. (PETRUCCI et al., 2006; BENEY; SÉRÉ, 2001).

\section{Metodología de trabajo}

La asignatura Física II se dicta en primer año de las carreras de Licenciatura y Profesorado en Física de la UNLPam, tiene una carga horaria de diez (10) horas semanales. Proyectamos nuestras actividades de manera integrada, donde las clases teóricas, los ejercicios 
de lápiz y papel y la experimentación se desarrollan en forma conjunta. Se trata de fomentar el desarrollo de actitudes y procedimientos en los alumnos que acompañen el aprendizaje significativo de conceptos. Entendiendo por tal, a aquel aprendizaje a partir del cual el estudiante es capaz de explicar y resolver por sí mismo distintas situaciones, que, en definitiva es cuando las comprende (MOREIRA; GRECA, 2003; GIL PÉREZ et al., 1999).

Dentro de las distintas actividades de aula y a lo largo de todo el curso, propusimos problemas cuya resolución requirió la discusión entre pares. El objetivo de estas actividades fue favorecer la integración y profundización de los conceptos involucrados. La mayoría de ellos fueron problemas ricos en contexto. Esta estrategia de enseñanza se fundamenta en el trabajo en grupos cooperativos y en su resolución el alumno se involucra directamente (HELLER; HELLER, 1999). Cada grupo de trabajo expuso sus resultados en el pizarrón.

En el caso de la unidad Sistemas Extensos y Movimiento de Rotación, se presentó este problema que prevé no sólo el análisis de la situación planteada sino también la observación y medición del movimiento de un carretel real.

Se dedicaron dos clases, con un total seis horas, a esta actividad. En la primera clase se desarrolló la parte experimental y en la siguiente la resolución analítica del problema planteado y la interpretación de los resultados obtenidos.

Se realizó una primera experiencia en el año 2014, lo que permitió algunos ajustes que fueron tenidos en cuenta en el año lectivo 2015. Relatamos aquí lo ocurrido en esta oportunidad. Cursaron la materia cuatro estudiantes, motivo por el cual se constituyó un único grupo de trabajo.

\section{Desarrollo de las clases}

En esta sección mostramos el protocolo de la guía de trabajo experimental y la solución de los estudiantes.

\section{FÍSICA II}

\section{Estudio de un carrete}

Se tiene un carrete (puede ser un yo-yo o uno comercial en el que vienen arrollados hilos, sedales o cables) y se desea estudiar cómo se moverá en algunas circunstancias de interés.

\section{Primera parte: Medición del momento de inercia del carrete}

Observen el carrete del que disponen ¿es geométricamente simple? ¿Creen que es posible calcular su momento de inercia a partir de su forma? ¿Qué deberían medir? Por supuesto, contamos con balanza, cintas métricas y calibres para realizar las mediciones necesarias. 
Si no podemos calcular su momento de inercia a partir de mediciones sencillas ¿habrá otra forma de medir I? Si creen que es posible, ¿cómo lo harían? Si es necesario realizar alguna experiencia, propongan un diseño que puedan llevar a cabo.

\section{Segunda parte: Estudio del movimiento del carrete cuando rueda sobre una superficie horizontal}

Se arrolla un hilo en el cilindro interior del carrete. Les pedimos que analicen su movimiento tanto analítica como experimentalmente cuando se lo hace rodar sin deslizamiento sobre una superficie horizontal tirando del hilo.

\section{Situación 1: El hilo está horizontal}

a) ¿Qué fuerzas actúan sobre el carrete? ¿cuál será su aceleración? Desarrollen las ecuaciones que necesiten para responder la pregunta.

b) ¿Cómo se moverá el carrete? ¿El hilo se enrolla o se desenrolla? ¿Hacia dónde apunta la fuerza de rozamiento?

c) ¿Será lo mismo si el hilo "sale por arriba" o "sale por abajo”?

\section{Situación 2: El hilo forma un ángulo $\boldsymbol{\theta}$ con la horizontal}

a) ¿Qué fuerzas actúan sobre el carrete? ¿cuál será su aceleración? Desarrollen las ecuaciones que necesiten para responder la pregunta. ¿Para qué lado apunta la fuerza de rozamiento?

b) ¿Cómo se moverá el carrete? ¿el hilo ser enrolla o se desenrolla? ¿En qué influye el ángulo $\theta$ ?

c) ¿Será lo mismo si el hilo "sale por arriba” o "sale por abajo”?

Analicen cuidadosamente los resultados analíticos e interprétenlos experimentando con el carrete.

Registren todo lo que hacen y elaboren un informe escrito.

El problema fue presentado a los alumnos la semana anterior al desarrollo de la experiencia, les dimos la guía y les mostramos el carretel con el que iban a trabajar. Les pedimos que pensaran, individualmente, distintas formas de medir el momento de inercia del carrete.

En la clase anterior a la destinada al laboratorio, nos abocamos a la discusión de las respuestas que habían traído pensadas para la Primera Parte. Generamos la discusión respecto al método con el que podían calcular el momento de inercia del carrete respecto de un eje que pase por su centro y tiene la dirección de su eje de simetría, dado que se lo haría girar en torno a ese eje. 
Los alumnos observaron detenidamente el carrete y se dieron cuenta de que los cilindros exteriores no eran uniformes lo que los llevó a pensar que para calcular su momento de inercia deberían calcular el de las distintas partes y hacer algunas suposiciones sobre el espesor del material para evaluar la masa, lo que no es sencillo. Tampoco sabían si el cilindro interior era macizo o hueco. Como consecuencia, concluyen que era necesario realizar un experimento dinámico para encontrar el valor del momento de inercia $(I)$.

Los profesores los animamos a realizar una estimación del orden de magnitud de I teniendo en cuenta que podía tener un valor próximo a $\frac{m R^{2}}{2}$. Midieron entonces la masa lo que arrojó $(32,8 \pm 0,1)$ g y el diámetro exterior, $\mathrm{R}=(4,50 \pm 0,02) \mathrm{cm}$, encontrando que $\mathrm{I} \approx 3,3 \mathrm{x}$ $10^{-5} \mathrm{~kg} \mathrm{~m}^{2}$.

En su argumentación sobre cómo desarrollar la experiencia para medir el momento de inercia, los estudiantes llegaron a la clase de laboratorio con dos propuestas: 1) hacer rodar el carrete por un plano inclinado y 2) hacerlo rodar por una superficie horizontal por efecto de un peso colgado en el extremo libre del hilo arrollado en el carrete. A partir de la discusión sobre cuál de las propuestas sería la más factible de llevar a cabo con los elementos de que disponían en el laboratorio, se decidieron por la primera opción.

Discutieron entre ellos el diseño experimental y procedieron a analizar la dinámica de la situación. Dibujaron el carrete de masa m, radio R y momento de inercia $I$, ubicado sobre el plano inclinado un ángulo $\theta$ respecto de la horizontal (Fig. 1).

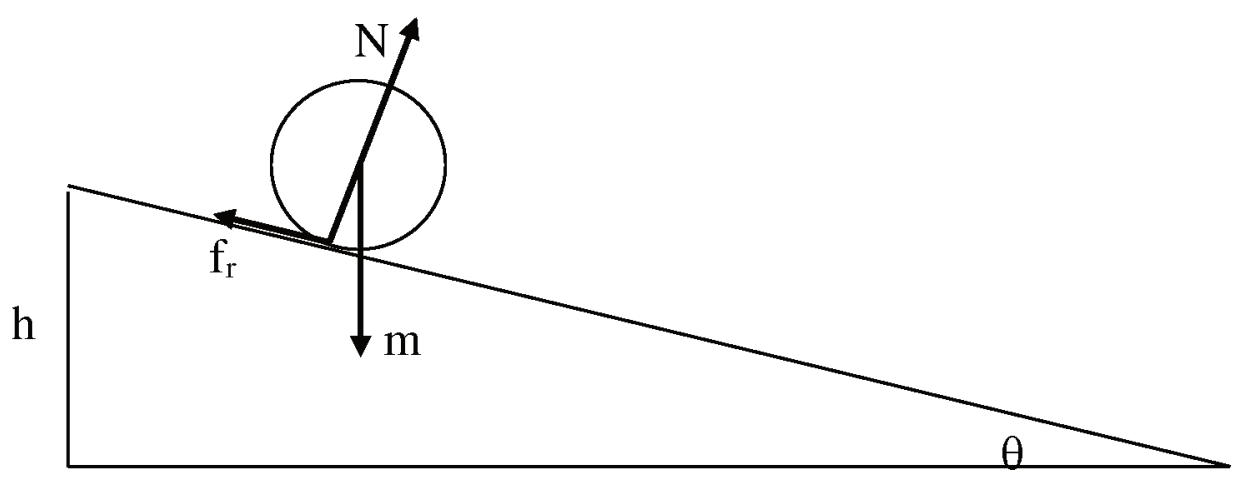

Fig. 1 -Esquema de la situación experimental propuesta por los estudiantes.

Identificaron las fuerzas que actúan y plantearon las ecuaciones para encontrar la expresión del momento de inercia del carrete en función de la aceleración del centro de masas:

$$
I=\frac{m(g \operatorname{sen} \theta-a) R^{2}}{a}
$$


Donde $a$ es la aceleración del CM del carrete y han aplicado la condición de rodadura: $\alpha=\frac{a}{R}$, donde $\alpha$ es la aceleración angular del carrete. Quedó claro entonces, que para evaluar el momento de inercia debían medir la aceleración $(a)$ del carrete.

Los docentes controlamos las conclusiones a las que habían arribado y los interrogamos sobre cómo harían para encontrar la aceleración. Proponen medir el tiempo que tarda en rodar el carrete desde el reposo una distancia D sobre el plano inclinado. A continuación reprodujeron la situación en el laboratorio; usaron una madera rugosa para asegurarse que el carrete rodara sin deslizar. Hicieron algunos intentos previos y sobre esta base decidieron que el ángulo de inclinación del plano debía ser pequeño. Practicaron hasta encontrar la manera de reducir el error experimental al mínimo para medir el tiempo. Como las medidas resultaron bastante dispersas, tomaron una muestra de 25 tiros.

Al llevar a cabo la experiencia obtuvimos un valor promedio para el tiempo de $(2,93 \pm 0,03) \mathrm{s}$. El valor medido de $\mathrm{D}$ es $(0,691 \pm 0,005) \mathrm{m}$. El error estimado se debe a la indeterminación en la posición inicial y la posición en la cual se aprieta al cronómetro al finalizar el trayecto. Entonces, $a=\frac{2 D}{\Delta t^{2}}=0,161 \frac{m}{s^{2}}$. También debemos calcular el error lo que arroja $\Delta a=0,004 \frac{m}{s^{2}}$.

Para hallar el valor del $\operatorname{sen} \theta$, se ayudaron con un nivel para medir la altura h con un calibre (ver Fig. 1) obteniendo h $=(1,832 \pm 0.004) \mathrm{cm}$.

Una vez que reemplazaron los valores medidos en la ecuación (1) y que hicieron los cálculos del error correspondientes, el resultado fue:

$$
\mathrm{I}=(4,1 \pm 0,4) \times 10^{-5} \mathrm{~kg} \mathrm{~m}^{2}
$$

En la clase siguiente se ocuparon de dar respuesta a la segunda parte de la guía. Luego de dibujar el esquema de fuerzas para cada una de las cuatro situaciones, se estableció una discusión sobre la elección del sistema de coordenadas ¿qué lado elegimos como positivo? En consonancia con esa elección, ¿cuál será el signo de giro positivo? ¿El de las agujas del reloj o el contrario? Finalmente plantearon las ecuaciones y las resolvieron. Todo el proceso tomó cerca de una hora.

En ese momento empezó la puesta en común. Cada alumno desarrolló en el pizarrón una de las situaciones, mientras el resto participaba y contribuía a la solución. En cada caso se los invitó a analizar los resultados y a experimentar con el carretel. No resultó obvia la relación entre el signo de la aceleración y el hecho de que el hilo se enrolle o desenrolle. Hicieron rodar el carrete con el hilo saliendo "por arriba" o "por abajo" y el comportamiento del hilo les pareció sorprendente. Los alumnos hicieron rodar el carrete tratando de reproducir las situaciones analizadas y observar si el hilo se enrollaba o desenrollaba.

Seguidamente mostramos brevemente la solución del problema, tal como la escribieron en el pizarrón. 


\section{1) Situación 1: El hilo está horizontal}

\section{a) El hilo sale "por arriba"}

Dibujaron la dirección de la fuerza de rozamiento hacia la izquierda y plantearon las ecuaciones de traslación y de rotación bajo la condición de que el carretel rueda sin deslizar. Eligieron un sistema de referencia positivo hacia la derecha para la traslación y, de acuerdo a eso, a positivo en la dirección de las agujas del reloj.

$$
\begin{gathered}
T-f_{r}=m a \\
T r+f_{r} R=I \alpha
\end{gathered}
$$

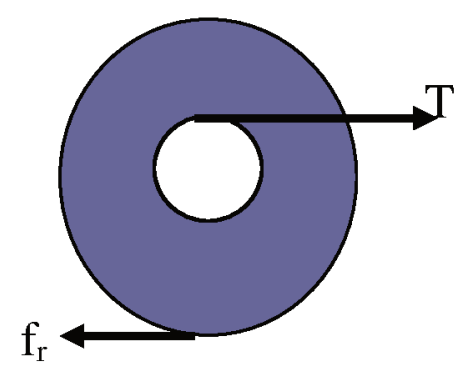

Fig. 2 - Esquema de fuerza para la situación la.
Dónde: $T$ es la tensión del hilo, $f_{r}$ la fuerza de rozamiento, $r$ el radio del cilindro interior sobre el que está arrollado el hilo y $R$ el radio de los cilindros exteriores sobre los que rueda el carretel; $m$ es la masa e $I$ el momento de inercia del carretel, $\alpha$ es la aceleración angular y $a$ la aceleración de su CM.

A estas ecuaciones se debe agregar la condición de rodadura: $a=\alpha R$.

La solución de este sistema de ecuaciones les dio como resultado:

$$
a=\frac{T R(r+R))}{I+m R^{2}} \quad \text { y } \quad f_{r}=\frac{T(I-m R r)}{\left(I+m R^{2}\right)}
$$

Analizaron las ecuaciones (3) y concluyeron que la aceleración siempre es positiva, es decir hacia la derecha en nuestro dibujo. Esto significa que el hilo se desenrolla (siempre suponiendo que parte del reposo).

Luego que analizaron este resultado procedieron a reproducir la situación para vivenciar el movimiento del mismo y poder observar si, efectivamente, éste coincidía con los resultados analíticos. Prestaron especial atención al hecho de que el hilo se desenrollaba.

$\mathrm{Al}$ analizar la fuerza de rozamiento se dieron cuenta de que puede ser hacia la izquierda (positiva si coincide con nuestra propuesta inicial) o hacia la derecha, según si $I$ es mayor o menor que el producto $m R r$. Los alumnos midieron el valor de los radios y encontraron que $\mathrm{R}=(4,50 \pm 0,02) \mathrm{cm} \quad$ y $\quad \mathrm{r}=(3,00 \pm 0,02) \mathrm{cm}$. Con el valor hallado en la clase anterior $\left(I=4,1 \times 10^{-5} \mathrm{~kg} \mathrm{~m}^{2}\right)$ encontraron que la fuerza de roce resultaba negativa, por lo tanto la misma apunta hacia la derecha en su dibujo. Esto fue hondamente analizado dado que experimentalmente no es posible observar que la dirección de esta fuerza es la del movimiento. Es necesario aclarar que este aspecto resulto relevante para estos alumnos, dado que en general 
solemos afirmar "la fuerza de roce siempre se opone al movimiento". Entonces debemos destacar que este experimento es importante para aclarar aspectos como este.

En la intervención que hicimos para ir aclarando todas estas ideas, reforzamos el hecho de que esta fuerza de roce es de carácter estático, por lo que su valor y dirección son los necesarios para que el carrete no deslice.

Una vez que los estudiantes terminaron con el análisis del movimiento para este carrete, los docentes los llevamos a realizar un análisis más general, es decir para cualquier otro carrete. Por ejemplo, ¿qué sucedería si el producto $m R r$ fuera menor que el momento de inercia $I$ ? en este caso $\mathrm{f}_{\mathrm{r}}$ es positiva (ecuación3) coincidiendo su sentido con el dibujado. Concluyeron que si el radio interior es menor, cambia la dirección de la fuerza de rozamiento.

b) EI hilo sale "por abajo"

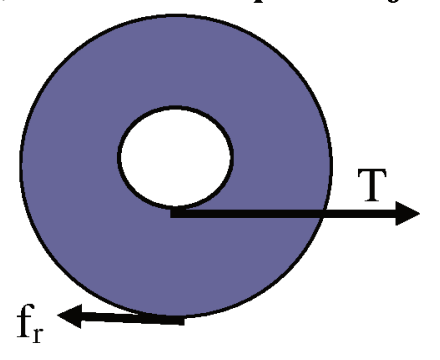

Fig. 2 - Esquema de fuerza para la situación $1 b$.

En este caso las ecuaciones planteadas fueron:

$$
\begin{aligned}
& T-f_{r}=m a \\
& \quad f_{r} R-T r=I \alpha
\end{aligned}
$$

Y la solución a la que llegaron fue:

$$
a=\frac{T R(R-r))}{I+m R^{2}} \quad \text { y } \quad f_{r}=\frac{T(I+m R r)}{\left(I+m R^{2}\right)}
$$

$\mathrm{Al}$ analizar el resultado tuvieron en cuenta que la condición inicial es que $\mathrm{r}<\mathrm{R}$, por lo que ambas cantidades siempre son positivas, la aceleración es hacia la derecha y el hilo siempre se enrolla. La fuerza de rozamiento es hacia la izquierda, como la dibujaron. También en este caso reprodujeron la situación para observar el movimiento del carrete y concluyeron que el hilo se enrolla.

\section{2) Situación 2: El hilo forma un ángulo $\theta$ con la horizontal}

Los docentes hicieron observar a los estudiantes que el hilo tiene una dirección tangente al borde del cilindro interior en el punto en el cual se separa de él, por lo cual la fuerza de tensión es perpendicular a r en ese punto. 
a) El hilo sale "por arriba"



Plantearon las ecuaciones:

$$
\begin{aligned}
& T \cos \theta-f_{r}=m a \\
& T r+f_{r} R=I \alpha
\end{aligned}
$$

Por lo que:

Fig. 4 - Esquema de fuerzas para la situación 2 a).

$$
a=\frac{T R(r+R \cos \theta))}{I+m R^{2}}
$$

$$
\text { y } f_{r}=\frac{T(I \cos \theta-m R r)}{\left(I+m R^{2}\right)}
$$

En su discusión, los alumnos concluyeron que la aceleración es siempre positiva, es decir, hacia la derecha por lo que el hilo de desenrolla. El signo de la fuerza de rozamiento depende ahora no sólo de la relación entre $\mathrm{R}$ y $\mathrm{r}$ sino que interviene $\theta$, tal que si

$$
(I \cos \theta-m R r)>0
$$

la fuerza de rozamiento es hacia la izquierda (como la dibujamos). Para el carrete que estaban utilizando, esta cantidad siempre es negativa, independientemente del ángulo y la fuerza de rozamiento es hacia la derecha (en su dibujo).

\section{b) El hilo sale "por debajo"}

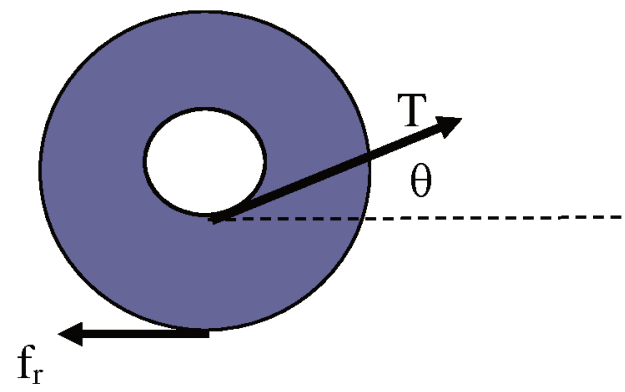

Fig. 5 - Esquema de fuerzas para la situación $2 b$ ).

$$
\begin{aligned}
& T \cos \theta-f_{r}=m a \\
& -T r+f_{r} R=I \alpha
\end{aligned}
$$

Entonces:

$$
\begin{aligned}
& a=\frac{T R(R \cos \theta-r))}{I+m R^{2}} \\
& \text { y } f_{r}=\frac{T(I \cos \theta+m R r)}{\left(I+m R^{2}\right)}
\end{aligned}
$$

En sus experiencias previas, los alumnos ya se habían dado cuenta que si el hilo sale por debajo, el movimiento del carrete es hacia adelante o hacia atrás según la inclinación del hilo. Inclusive habían observado que para un ángulo particular no rodaba. Al analizar las ecuaciones encontraron una confirmación y justificación para sus observaciones ya que la aceleración puede ser positiva o negativa según el valor de $\theta$. 
Si $\cos \theta<\frac{r}{R}$ la aceleración resulta negativa (hacia la izquierda) y el hilo se desenrolla.

En cuanto a la fuerza de rozamiento concluyeron que siempre es positiva, es decir hacia la izquierda (hacia atrás), como habían planteado.


Fig. 6 - Izquierda: Dibujo del carrete en la situación en que la aceleración es cero. Derecha. Fotografía del carrete en esa situación.

Para verificar lo que se demuestra a partir del análisis de las ecuaciones (9) los docentes guiamos a los estudiantes para que realizaran un gráfico similar al de la Figura (6). En ella se muestra que cuando se cumple la condición $\cos \theta=\frac{r}{R}$ la recta que tiene la dirección del hilo pasa por el punto de apoyo del carrete y el torque respecto a ese punto es cero. En este caso la aceleración es cero y el sistema se encuentra en una situación de equilibrio inestable. Fotografiaron el carrete para esta situación y midieron el ángulo $\theta$ sobre la foto, lo que incluyeron en el informe (Figura (6). Para su carrete, $\theta=48^{\circ}$.

Cada clase duró unas tres horas y los alumnos elaboraron un informe escrito que entregaron una semana después.

\section{Conclusiones}

La medición del momento de inercia del carrete enfrenta a los alumnos a una situación en la que no pueden aplicar los modelos sencillos que aparecen en los ejercicios y problemas de fin de capítulo de los textos (RESNICK et al., 2004; SEARS et al., 2004; SERWAY et al., 2009; TIPLER; MOSCA, 2010). En la mayoría de ellos los objetos que giran o ruedan son cilindros o esferas. Cuando el objeto es irregular (una calesita, por ejemplo) habitualmente se da su momento de inercia como un dato del ejercicio. La necesidad de cuestionarse el modelo y aceptar que es necesaria una forma indirecta de medición es enriquecedora y los obliga a 
proponer un método experimental. El diseño de experiencias sencillas exige no sólo imaginar un procedimiento sino ceñirse a los elementos de los cuales se dispone en el laboratorio y la elección de la forma más sencilla entre varias posibles. También deben considerar los errores de medición para estimar cuantas medidas tienen que realizar, cuales variables deben medirse con mayor cuidado y la evaluación de la incerteza del resultado alcanzado.

La situación planteada en la segunda parte ofreció la posibilidad de analizar distintos aspectos acerca de la rodadura y del movimiento de rotación en general que habían pasado desapercibidos a pesar de que se resolvieron una serie de ejercicios. La dinámica de rotaciones es un tema complejo y los alumnos pueden llegar a soluciones correctas sin darse cuenta de algunas cuestiones. El papel del rozamiento estático no es obvio y creemos que este problema ayuda a su comprensión.

Durante la discusión de los resultados se buscó que los estudiantes los interpretaran a partir de la aplicación de las ecuaciones. La correlación entre las ecuaciones, el movimiento posible y la dirección de la fuerza de rozamiento los ayudó a comprender el papel de la matemática en la resolución e interpretación de un problema físico. El ejercicio los llevó también a realizar experiencias sin una guía establecida por lo que debieron diseñarlas ellos mismos. Creemos que todo esto contribuye a la formación de sujetos independientes capaces de concretar experiencias e hipotetizar posibles resultados.

El hecho de hacer deducciones simbólicas, de tener que evaluar lo que ocurre al cambiar alguna variable y predecir resultados sin mediciones favorecería el desarrollo del pensamiento lógico y el análisis de la situación de manera más general como deberán hacer cada vez con más frecuencia al avanzar en sus carreras.

Las ventajas de aplicar esta propuesta frente a otras utilizadas en años anteriores se deben analizar más profundamente, lo que excede el propósito de este trabajo en el cual pretendemos plantear la propuesta didáctica.

\section{Bibliografía}

BENEY, M.; SÉRÉ, M. G. Entre réussir et comprendre ou de l'effet des consignes opératoires sur la compréhension des procédures de mesurage en TP de physique de 1r. cycle universitaire. Didaskalia, v. 19, p. 9-37. 2001.

COSTAMAGNA, A. El valor de la metaevaluación del cambio conceptual: una experiencia didáctica. Enseñanza de las Ciencias, v. 23, n. 3, p. 419-430, 2005.

DAVINI, M. C. Métodos de enseñanza. Didáctica general para maestros y profesores. Buenos Aires: Santillana, 2009. 239 p.

GIL PÉREZ, D.; FURIÓ MAS, C.; VALDÉS, P.; SALINAS, J.; MARTÍNEZ-TORREGROSA, J.; GUISASOLA, J.; GONZÁLEZ, E.; DUMAS-CARRÉ, A.; GOFFARD, M.; CARVALHO, A. M. P. ¿Tiene sentido seguir distinguiendo entre aprendizaje de conceptos, resolución de 
problemas de lápiz y papel y realización de prácticas de laboratorio? Enseñanza de las Ciencias, v. 17, n. 2, p. 311-320, 1999.

HELleR, K.; HELleR, P. Cooperative Group Problem Solving in Physics. Illinois: University of Minnessota, 1999. 172 p.

LITWIN, E. El oficio de enseñar. Condiciones y contextos. Buenos Aires: Paidós, 2009. 232 p.

MOREIRA, M. A.; GRECA, I. M. Cambio conceptual: análisis crítico y propuestas a la luz de la Teoría del Aprendizaje Significativo. Ciência \& Educação, v. 9, n. 2, p. 301-315, 2003.

PETRUCCI, D.; URE, J.; SALOMONE, H. Cómo ven a los trabajos prácticos de laboratorio de física los estudiantes universitarios. Revista de Enseñanza de la Física, v. 19, n. 1, p. 7-20, 2006.

REDISH, E. Teaching Physics with the Physics Suite. USA: Wiley \& Sons, INC, 2003. 216 p.

RESNICK, R.; HALLIDAY, D.; KRANE, K. Física. México: Compañía Editorial Continental, 2004. 566 p. v. 1.

SEARS, F.; FREEDMAN, M.; YOUNG, H.; ZEMANSKY, M. Física Universitaria. México: Pearson Education, 2004. 708 p. v. I.

SEWAY, R.; JEWET, J. Física para Ciencias e Ingeniería con Física Moderna. México: Cengage Learning Editores, 2009. 640 p. v. 1.

SOUTO, M. La clase escolar. Una mirada desde la didáctica de lo grupal. En: CAMILLONI y otros. Corrientes didácticas contemporáneas. Buenos Aires: Paidós, 1996. cap. 5. p. 117167.

TIPLER, P. A.; MOSCA, G. Física para la Ciencia y la Tecnología. Barcelona: Reverté, 2010. 692 p. v. I. 